\title{
The vibrational auto-adjusting perturbation theory (VAPT) $\ddagger$
}

\author{
Eduard Matito, ${ }^{1,} *$ Juan Manuel Barroso, ${ }^{2}$ Emili Besalú, ${ }^{2}$ Ove Christiansen, ${ }^{1}$ and Josep M. Luis ${ }^{2}, \dagger$ \\ 1 The Lundbeck Foundation Center for Theoretical Chemistry, \\ Department of Chemistry, University of Aarhus, Denmark \\ ${ }^{2}$ Institute of Computational Chemistry, Department of Chemistry, University of Girona
}

(Dated: November 20, 2008)

\begin{abstract}
In this work a new method to calculate anharmonic vibrational ground and excited state energies is proposed. The method relies on the auto-adjusting perturbation theory (APT) which has been successfully used to diagonalize square matrices. We use as zeroth order correction the self-consistent vibrational energies, and with the APT approach we calculate the vibrational anharmonic correlation correction to any desired order. In this paper we present the methodology and apply it to a model system and formaldehyde. Vibrational APT approach shows a robust convergent behavior even for the states where the standard (Rayleigh-Schrödinger) vibrational Møller-Plesset perturbation theory is clearly divergent.
\end{abstract}

\section{INTRODUCTION}

The calculation of vibrational spectrum of polyatomic molecules is not always an easy task. While small molecules allow for the calculation of anharmonic energies with great accuracy, as soon as the size of the molecule increases more and more approximations need to be taken into account in order to make the calculation computationally feasible. Assuming overall Born-Oppenheimer separation of electronic and nuclei motions [1], a first group of approximations concerns the accuracy of the electronic structure calculations and the potential energy surfaces (PESs) constructed from these calculations [2-4]. Here we concentrate on a second kind of approximations, those related with the method chosen to solve the vibrational Schrödinger equation.

The calculation of anharmonic energies can be done in a number of ways. One of the simplest of these methods, is the vibrational self-consistent field approach (VSCF) of Bowman [5-8]. In the VSCF approach a Hartree product of one-mode functions (denoted modals) is determined self-consistently by the application of the variational principle. Vibrational Møller-Plesset (VMP) [9-11], is to VSCF what MP [12] is to SCF in electronic structure theory. It is thus the simplest way to introduce correlation between modes. VMP2 usually provides reasonably accurate fundamental vibrational frequencies, and has been applied to many systems in its simplest two-mode coupling variant by Gerber and coworkers $[9,10]$. A number of developments aiming at rather large molecules has also recently been presented [13-15]. However, as shown by one of us [11], VMP has other severe problems, e.g. handling systems with Fermi resonance. Vibrational Coupled-Cluster (VCC) $[16,17]$ and vibrational configuration interactions (VCI) [18-21] are good alternatives to VMP calculations which can cope more effectively with Fermi resonance. VCI calculations are computationally more demanding as compared with low-order corrections of VMP series. VCC methods, when implemented in a straightforward fashion, scale even worse than VCI with respect to the number of modes. However, efficient implementation of VCC methods holds promise of accurate results at a reasonable computational time. Recently a VCC[2] method for a two-mode coupled Hamiltonian scaling as the corresponding VCI[2] method was reported [13], thus providing with higher correlation between mode pairs at similar computational expense as VCI.

While an efficient implementation of a general $\operatorname{VCC}[n]$ method is yet a challenge, a method which is easy to program and can cope with Fermi resonance would be desirable. In this line, the quasi-degenerate perturbation theory (QDPT) has been studied using as a starting either harmonic oscillator [22, 23] or the VSCF modals [24]. In this paper we present a new alternative to deal with resonant states based on the auto-adjusting perturbation theory (APT) [25]. The method, which has been used to diagonalize large matrices [26, 27], it is tested here as an alternative to Rayleigh Schrödinger perturbation theory (RSPT) in vibrational theory. Despite APT was not originally designed to deal with nearly degenerate states, it will be shown to be able to handle this kind of systems. Our vibrational APT (VAPT) implementation is based on a VSCF reference wavefunction, though any other reference wavefunction is possible. The VAPT approach is applied to two examples with well-known Fermi resonance problems: $\mathrm{H}_{2} \mathrm{CO}$

\footnotetext{
$¥$ Dedicated to Prof. Santiago Olivella on occasion of his $65^{t h}$ birthday.
} 
and the model developed by Thompson and Truhlar [28]. Whereas RSPT-VMP methods do not converge for the calculation of some states, APT-VMP is shown to converge in all cases, including the quasi-degenerate vibrational states for which standard (Rayleigh-Schrödinger) vibrational Møller-Plesset perturbation theory is clearly divergent. The manuscript is organized as follows: in the next section we briefly review VSCF and VMP methods and introduce VAPT. The section following describes the systems under study and the section afterwards presents the results. The paper concludes with the summary.

\section{METHODOLOGY}

We will here use the notation introduced elsewhere [29]. Capital letters refer to dimensions, while lower case letters are used for the dummy indexes. Hence, the number of degrees of freedom (the number of modes) is denoted as $M$, whereas $m$ corresponds to the index for the modes. $\phi_{s^{m}}^{m}\left(q_{m}\right)$ is used to denote the modals or the one-mode functions, indexed by $s^{m}$ for mode $m$ which depends on normal coordinate $q_{m}$. The dimension of the basis for mode $m$ is $N^{m}$ and the set of basis is assumed orthonormal so that $\left\langle\phi_{s^{m}}^{m}\left(q_{m}\right) \mid \phi_{r^{m}}^{m}\left(q_{m}\right)\right\rangle=\delta_{r^{m}, s^{m}}$.

In the following we will consider $M$-mode wavefunctions as a Hartree product of modals:

$$
\left|\Phi_{s}(\mathbf{q})\right\rangle=\prod_{m=1}^{M} \phi_{s^{m}}^{m}\left(q_{m}\right)=\phi_{s^{1}}^{1}\left(q_{1}\right) \phi_{s^{2}}^{2}\left(q_{2}\right) \ldots \phi_{s^{M}}^{M}\left(q_{M}\right)
$$

Any wavefunction in the $M$-mode space can be written as a linear combination of Hartree products, $\left|\Phi_{c}(\mathbf{q})\right\rangle=\sum_{s} c_{s}\left|\Phi_{s}(\mathbf{q})\right\rangle$. Thus, the exact solution for the vibrational wavefunction can be obtained for a complete modal set $\left(N^{m} \rightarrow \infty\right)$ and a complete expansion of Hartree products. Obviously, the exact solution to the nuclear Schrödinger equation can not be obtained in general, and several approximations need to be taken into account to solve the problem.

As commonly done in the literature, we will neglect rotovibrational couplings and mass-dependent terms in the effective vibrational potential of the full Watson Hamiltonian [30, 31]:

$$
H=-\frac{1}{2} \sum_{m=1}^{M} \frac{\partial^{2}}{\partial q_{m}^{2}}+V(\mathbf{q})
$$

The potential function, $V(\mathbf{q})$, usually consists of a linear combination of products of normal coordinates, i.e., a polynomial in powers of $q_{s}^{m}$. Therefore, the Hamiltonian becomes a sum over $T$ product terms:

$$
H=\sum_{t=1}^{T} \prod_{m}^{M} h^{m, t}
$$

and the integrals to be solved only involve one coordinate at a time $[32,33]$.

\section{Vibrational self-consistent field (VSCF) theory}

In the VSCF method we variationally optimize the energy:

$$
e_{s}=\left\langle\Phi_{s}|H| \Phi_{s}\right\rangle
$$

under variation of the orthonormal modals. This condition leads straightforwardly to self-consistent field equations for the modals:

$$
F^{m, s} \phi_{s^{m}}^{m}=\epsilon_{s^{m}}^{m} \phi_{s^{m}}^{m}
$$

where the mean field Hamiltonian is given by:

$$
F^{m, s}=\left\langle\prod_{m^{\prime} \neq m}^{M} \phi_{s^{m^{\prime}}}^{m^{\prime}}|H| \prod_{m^{\prime \prime} \neq m}^{M} \phi_{s^{m^{\prime \prime}}}^{m^{\prime \prime}}\right\rangle
$$


Due to the fact modes are distinguishable, VSCF can be performed analogously for any vibrational excited state. Since $H$ depends on $V(\mathbf{q})$ which contains the information of several modes, the mean field Hamiltonian depends implicitly on the other modals. Hence the Eq. 5 needs to be solved iteratively. Once self-consistency is achieved a set of variationally optimized modals and modals energies are obtained for the ground state.

Although some coupling between the modes is implicitly included by this mean field approach, explicit mode correlation is ignored. The set of virtual modes obtained can be used in post-VSCF methods to introduce explicit mode correlation. One might mention here that resonances are troublesome for VSCF. Resonance in polyatomic molecules can occur when a given vibrational mode has the same energy as another level associated with another vibration or a combination of vibrations. In that event, more than a single Hartree product is essential to properly describe the system.

\section{Vibrational Møller-Plesset (VMP) Theory}

In the VMP [9-11] theory, as in the case of the MP, the zeroth order Hamiltonian is obtained from the VSCF approach. Let us here separate the Hamiltonian as follows:

$$
H=H^{(0)}+V=\sum_{m} F^{m, s}+V
$$

where $V$, the perturbation, is called fluctuation potential. Expanding the energy and the wavefunction in orders of the perturbation we get:

$$
\begin{gathered}
e=\sum_{i=0}^{\infty} e^{(i)} \\
|\Psi\rangle=\sum_{i=0}^{\infty}\left|\Psi^{(i)}\right\rangle
\end{gathered}
$$

Using VSCF as zeroth order wavefunction (i.e. $\left|\Psi^{(0)}\right\rangle=\left|\Phi_{s}\right\rangle$ ), and assuming intermediate normalization, $\left\langle\Psi^{(0)} \mid \Psi^{(n)}\right\rangle=\delta_{0, n}$, we get from the zeroth and the first order equations:

$$
e_{s, \mathrm{VSCF}}=\left\langle\Psi^{(0)}\left|H^{(0)}\right| \Psi^{(0)}\right\rangle+\left\langle\Psi^{(0)}|V| \Psi^{(0)}\right\rangle=e^{(0)}+e^{(1)}
$$

Collecting terms of the same order in the perturbation expansion, the $n^{\text {th }}$ order equation is obtained:

$$
\left(H^{(0)}-e^{(0)}\right)\left|\Psi^{(n)}\right\rangle=-V\left|\Psi^{(n-1)}\right\rangle+\sum_{k=1}^{n} e^{(k)}\left|\Psi^{(n-k)}\right\rangle
$$

The sigma vector is obtained by projection against the lower order correction to the wavefunction:

$$
\left|\sigma^{(k)}\right\rangle=H\left|\Psi^{(k-1)}\right\rangle
$$

We get the energy and the wavefunction correction in terms of $\left|\sigma^{(n)}\right\rangle$ :

$$
\begin{aligned}
e^{(n)} & =\left\langle\Psi^{(0)} \mid \sigma^{(n)}\right\rangle \quad \forall n>1 \\
\left|\Psi^{(n)}\right\rangle & =\left(H^{(0)}-e^{(0)}\right)^{-1}\left(H^{(0)}\left|\Psi^{(n-1)}\right\rangle-\left|\sigma^{(n)}\right\rangle+\sum_{k=1}^{n} e^{(k)}\left|\Psi^{(n-k)}\right\rangle\right)
\end{aligned}
$$

The calculation of VMP series is driven by the computation of the sigma vector. We can drastically reduce the computational cost for the energies, by means of the $2 n+1$ rule, which states that the correction $2 n+1$ for the 
energy can be calculated from the $n^{\text {th }}$-order and lower orders corrections to the wavefunction. Thus at the cost of $\left|\sigma^{(n+1)}\right\rangle$, we may get the $(2 n+1)^{t h}$ correction to the energy.

Although VMP method introduces explicit mode-correlation, basically it aims at introducing dynamic correlation i.e., interaction between non-degenerate states. Indeed, systems which contain considerable static correlation (Fermi resonance) usually produce divergent $\mathrm{VMP}[n]$ series [11]. It has also been illustrated that divergent VMP series are frequently found yet in the non-degenerate case.

In the next subsection we introduce the vibrational auto-adjusting perturbation theory, which by a two-step procedure, repeated at each order, provides energy and wavefunction corrections. The procedure consists of a perturbative correction and a transformation of the system on the basis of this perturbative correction. This dynamic reformulation of the system is what makes the APT different from common RSPT, makes possible the treatment of static correlation and will overall significantly modify the behavior of the perturbation series.

\section{Vibrational auto-adjusting perturbation theory (VAPT)}

Besalú et al. [25] described the APT as an iterative procedure to diagonalize square matrices. The procedure is thus useful to solve CI problems and by collecting different orders, APT becomes an alternative perturbation theory to the traditional Rayleigh-Schrödinger one.

The APT starts its iterative procedure from an unperturbed system, $\left(H^{(0)}, \Psi^{(0)}\right)$ and gets a correction of its eigenvector and eigenvalue. In practice, the eigenvalue correction is chosen in a convenient way to simplify the whole procedure, c.f. Ref. [25]. At the end of each step, a new unperturbed system is defined from the corrected eigenvector and eigenvalue. A new iteration step involves a new perturbation correction of the new eigenvalue and eigenvector [25].

The main distinctive feature of APT is that it relaxes the system by re-defining the unperturbed system after each perturbation step. This is what makes APT capable of handling quasi-degeneracy of the eigenstates in a better way than RSPT. It does this in a black-box way, avoiding a priori choice of modals and configurations.

In this paper, the APT is used to introduce mode-correlation between VSCF modals. Using the VSCF as unperturbed system, and RSPT as the perturbation method to get a first order correction of the eigenvector and a second order correction of the eigenvalue, we obtain the APT $[n]$ series, which in analogy to other vibrational theories we will call $\operatorname{VAPT}[n]$.

The wavefunction and the energy for the VAPT series are calculated in an analogous way to RSPT theory. Assuming convergence:

$$
\begin{aligned}
e=\lim _{n \rightarrow \infty} \bar{e}_{\mathrm{VAPT}}^{(n)} & \bar{e}_{\mathrm{VAPT}}^{(n)}=\sum_{k=0}^{n} e_{\mathrm{VAPT}}^{(k)} \\
|\Psi\rangle=\lim _{n \rightarrow \infty}\left|\bar{\Psi}_{\mathrm{VAPT}}^{(n)}\right\rangle & \left|\bar{\Psi}_{\mathrm{VAPT}}^{(n)}\right\rangle=\sum_{k=0}^{n}\left|\Psi_{\mathrm{VAPT}}^{(k)}\right\rangle
\end{aligned}
$$

Instead of using all corrections up to a given order, in APT we work with the energy corrected up to the target order, i.e., the accumulated correction (accumulated quantities are denoted with an overline). This is a feature which makes APT implementation quite appealing; while in RSPT all lower order energy and wavefunction corrections need to be stored, in APT we only need to store the last accumulated correction to compute the next one. The wavefunction corrected up to $n^{\text {th }}$ order is calculated as follows:

$$
\left|\bar{\Psi}_{\mathrm{VAPT}}^{(n)}\right\rangle=\left(\bar{e}_{\mathrm{VAPT}}^{(n-1)}-\bar{E}_{\mathrm{VAPT}}^{(n-1)}\right)^{-1}\left(\left|\bar{\sigma}_{\mathrm{VAPT}}^{(n-1)}\right\rangle-\bar{E}_{\mathrm{VAPT}}^{(n-1)}\left|\bar{\Psi}_{\mathrm{VAPT}}^{(n-1)}\right\rangle\right)
$$

where :

$$
\begin{aligned}
\left|\bar{\sigma}_{\mathrm{VAPT}}^{(n)}\right\rangle & =H\left|\bar{\Psi}_{\mathrm{VAPT}}^{(n)}\right\rangle \\
\bar{e}_{\mathrm{VAPT}}^{(n)} & =\left\langle\Psi^{(0)} \mid \sigma_{\mathrm{VAPT}}^{(n)}\right\rangle
\end{aligned}
$$


where we have used lower case for the energy to emphasize it is a scalar, corresponding to the $s^{\text {th }}$ state we are studying. When capitals are used for the energy, we refer to a diagonal matrix containing the energy correction for all states. Notice, however, that unlike VMP $[n]$, VAPT requires the calculation of the wavefunction before subsequently using it in the calculation of the energy correction corresponding to that wavefunction. Because of this, there is no $2 n+1$ rule for VAPT. In addition, to compute the $n^{t h}$ order wavefunction, one needs to calculate the $\bar{E}_{\mathrm{VAPT}}^{(n-1)}$ which is a diagonal matrix containing the effect of the correction on the other states $s \neq i$ :

$$
\bar{E}_{i i}^{(n)}=H_{i i}-H_{i s} \bar{\Psi}_{i s}^{(n)} \quad \forall i \neq s
$$

Notice, that APT needs to store the $s^{\text {th }}$ column and the diagonal of $\mathrm{H}$, the accumulated eigenvalue correction, $\left|\Psi^{(n)}\right\rangle$ and $\sigma^{(n)}$. $E_{\text {VAPT }}^{(n-1)}$ can be calculated straightforwardly on-the-fly from previous quantities. Actually, comparing with Eq. 13, we see that APT $[n]$ bears a similar computational cost to $\operatorname{VMP}[n]$ (when the $2 n+1$ rule is not used for $\mathrm{VMP}$ ), and that the bottleneck of the algorithm is likewise the calculation of the sigma vector.

Since we cannot benefit from the $2 n+1$ rule, the cost of VAPT is about twice the cost of VMP. One should also notice that since the MP partition is not kept during the iteration procedure, VAPT is not size-extensive. Although these may seem important drawbacks against VAPT, there are several other features that compensate. In the VAPT hereof, we have chosen RSPT first order correction of the wavefunction as the first order correction of the wavefunction in VAPT. In addition, the first-order correction of the energy in VAPT is taken as the first plus the second order corrections of RSPT. Therefore, the energy corrected up to first order with VAPT corresponds to the energy corrected up to second order with RSPT. It is thus expected that VAPT $[n]$ introduces more correlation than $\operatorname{VMP}[n]$. Another advantage of APT, is that it redefines the unperturbed system in every iteration. Although it is somehow hidden in the previous equations this property is responsible for the ability of VAPT to deal with quasi-degenerate systems. Altogether, higher cost of VAPT pays off by getting more accurate results than the corresponding order in the VMP series (vide infra) and providing convergent series in case of strong resonance.

\section{COMPUTATIONAL DETAILS}

The calculations are based on those done in Ref. [11] on formaldehyde and 2DB, a two-mode model system developed by Thompson and Truhlar [28]. 2DB was developed with the aim to have a simple model which displays Fermi resonance and is represented with the Hamiltonian:

$$
H=-\frac{1}{2} \frac{\partial^{2}}{\partial q_{1}^{2}}+\frac{1}{2} \omega_{1}^{2} q_{1}^{2}-\frac{1}{2} \frac{\partial^{2}}{\partial q_{2}^{2}}+\frac{1}{2} \omega_{2}^{2} q_{2}^{2}+k_{122} q_{1} q_{2}^{2}+k_{111} q_{1}^{3}
$$

where $w_{1}=1.4, w_{2}=0.7, k_{122}=-0.8$ and $k_{111}=0.0064$. By construction $w_{1}=2 w_{2}$ and coupling between the modes $\left(k_{122}\right)$ is significant; these are the reasons for the system to exhibit Fermi resonance. We have used a basis set of 33 harmonic oscillators. FVCI calculations have been performed using the full basis. We note in passing that the FVCI results for 2DB model in Ref. [11] are not in this basis as indicated erratically, but in a smaller basis of 7 harmonic oscillators. The differences are minor, in the worst case affecting the fourth digit, and by no means have any effect on the conclusions drawn in the paper.

Formaldehyde calculations are based on the quartic force field of Romanowski et al. [34] using 7 harmonic oscillators as the basis set for the VSCF calculation, 4 of which were retained for the VCI[3] and VMP $[n]$ calculations. At this level of theory the same divergent character of the $\operatorname{VMP}[n]$ series found by Christiansen [11] using 7 modals for FVCI calculations is reproduced for 3 of the 4 strongly divergent series. Although we have reduced the number of divergent cases at our disposal to test VAPT method, we believe these strongly divergent cases in formaldehyde and those found in the 2DB model are sufficient to illustrate the performance of VAPT. The reason for this simplification of the correlated calculation is practical. At this stage the VAPT $[n]$ series is calculated with the VAPT program [35] by using the (F)VCI matrix generated with MidasCpp [36]. Reducing the number of correlated modals and the number of excitations we reduce the size of the VCI matrix which needs to be stored on disk. Since the only purpose of this paper is to test VAPT for VMP divergent series we believe this simplification has no effect on the conclusions drawn in this manuscript. 
FIG. 1: Difference between VMP $[n]$ and FVCI energies for the model 2DB.

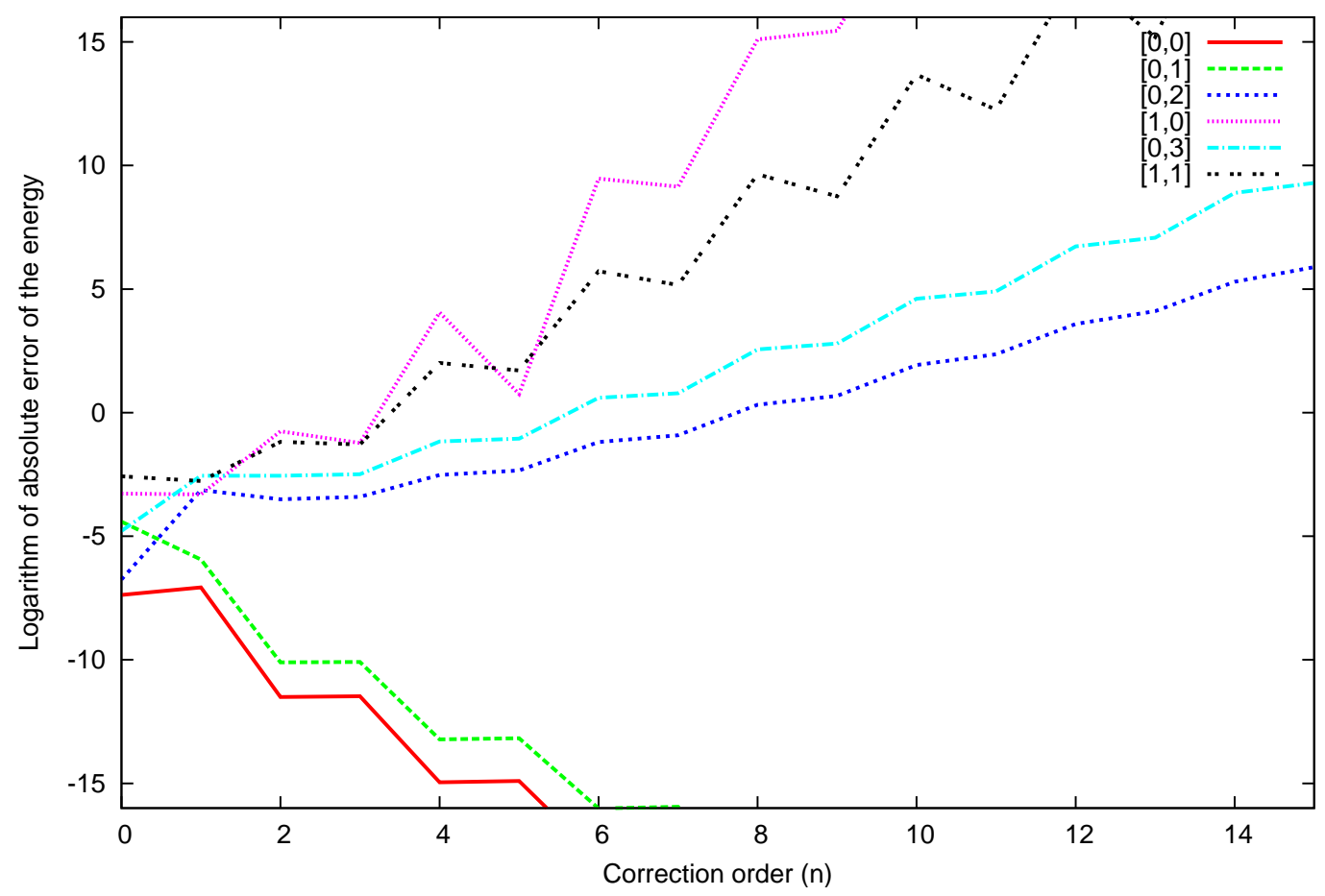

RESULTS

2DB model

Figure 1 summarizes the VMP results for some selected states; each calculation has been performed using the modals optimized for the specific state ( $s$ hereafter). From the chosen set, four out of six states produce divergent VMP series, as already reported [11]. In the supporting information (SI), the reader may find the tables with these results and also the calculations performed using ground-state $(g s)$ modals for all states. In addition, the SI collects the tables with the calculations using Epstein-Nesbet (EN) partition. All the results show the same divergent trends for the same states, regardless the partition or the modals used for the calculation.

In Figure 2 we give the energies for this set of states calculated using VAPT. The results are likewise calculated using Møller-Plesset partition and the ss modals. Indeed, as it was explained in the previous section, in the VAPT procedure, the user-defined partition (here Møller-Plesset) is only used in the first iteration. In the second and subsequent iterations the partition is dynamically redefined by the VAPT method. It is shown how VAPT converges the energy up to $10^{\text {th }}$ digit for all states with less than 15 iterations, and most of them in less than 8 iterations. Only $[1,0]$ and $[1,1]$ present slow convergent series. In the SI one may find tables for these calculations using MP and EN partitions, as well as working with $s s$ and $g s$ modals. The same qualitative results are reproduced, irrespective of the partition and the modals used for the calculation, excepting $g s$-EN-VAPT which converges the VAPT series for $[1,1]$ in the wrong state, $[0,3]$. Although VAPT never diverges in these examples, when the zeroth order wavefunction is not good enough it can fail in targeting the correct state. It is worth mentioning the outperforming role of VAPT[4], which gives results converged up to $7^{\text {th }}$ decimal place for all states but $[1,1]$. 
FIG. 2: Difference between VAPT $[n]$ and FVCI energies for the model 2DB.

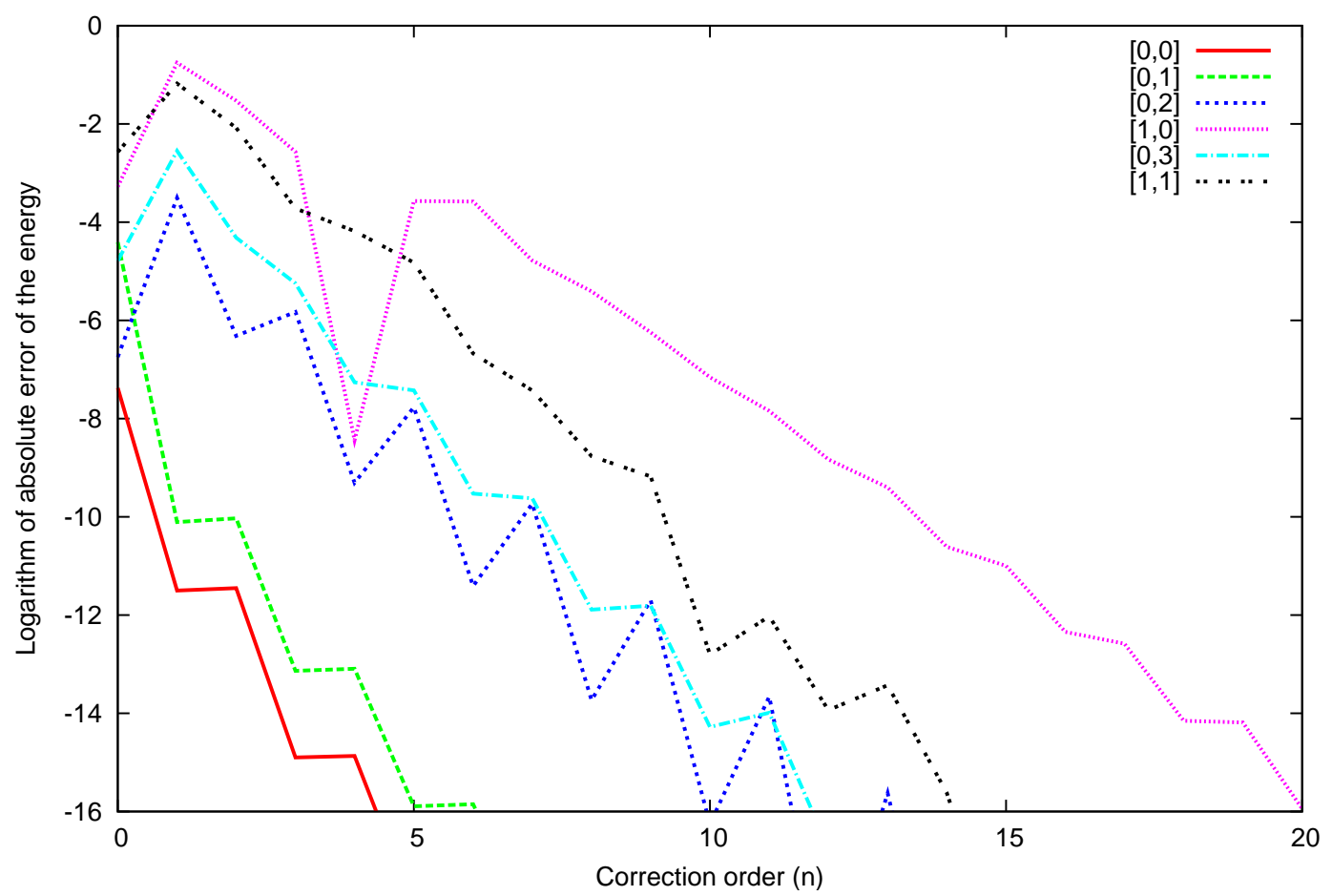

Formaldehyde

Table I collects the fundamental transition energies for formaldehyde computed with VMP and VAPT methods. One may observe that VMP diverges for the state $[0,0,0,0,1,0]$, while VAPT series is convergent for this state. In Table II we give overtones and combination bands below $9000 \mathrm{~cm}^{-1}$. We can clearly see two states with divergent VMP series, $[0,0,1,0,0,1]$ and $[0,1,0,0,0,1]$. Both are converged using VAPT, though the latter converges to the wrong state, $[0,0,0,0,1,0]$, which was the troublesome fundamental. This puts forward the reason for the divergent series for these two states, $[0,0,0,0,1,0]$ and $[0,1,0,0,0,1]$, with RSPT: there is high coupling between them. Although this is beyond the scope of this paper, it is worth mentioning there are ways to target the right state by tuning the perturbation. For instance, it can be imposed that the highest contribution from a given Hartree product is preserved in the iteration procedure. Research in this line is underway in our groups.

For the non-troublesome states of formaldehyde (those not divergent in RSPT), VMP[2] provides an accuracy of $5 \mathrm{~cm}^{-1}$, an indication that performance of low-order corrections of VMP acquire a good compromise between accuracy and computational cost. VAPT[1] energy is, in the present version of VAPT, equal to VMP[2], and it is thus not surprising that VAPT[2] performs similar or better, even for troublesome states. One can also observe than VAPT[4] results are less that $4 \mathrm{~cm}^{-1}$ off the VCI[3] results, even for the troublesome states (the only exception is, of course, the state which is not correctly targeted with VAPT).

In the SI the reader may find the tables for formaldehyde $g s$ and $s s$ calculations with both EP and MP partitions for VMP and VAPT series. $s s$-EN-VAPT results, $g s$-EN-VAPT and $g s$-MP-VAPT are similar to those presented for $s s$-MP-VAPT given in tables I and II. On the other hand, while $g s$-MP-RSPT results mimic those presented in the tables for ss-MP-RSPT results, when using EN partition (irrespective of the modals used for the calculation) the perturbation series for the state $[0,0,1,0,0,1]$ is convergent (though very slowly convergent for $g s$-EN-RSPT). Despite this particular success of $s s$-EN-RSPT, it is expected to find far more RSPT diverging cases in vibrational high-energy region, where Fermi resonances are common. Inasmuch EN-RSPT exhibited non-convergent series in the model system studied in this paper, we believe this particular example does not prove much about the performance of this 
TABLE I: Above the difference between VMP $[n]$ (specific-state) and VCI[3] energies for fundamental vibrations of formaldehyde. Below difference between VAPT $[n]$ (Møller-Plesset, specific-state) and VCI[3] energies for fundamental vibrations of formaldehyde. Units are $\mathrm{cm}^{-1}$.

\begin{tabular}{|c|c|c|c|c|c|c|c|}
\hline$n$ & {$[0,0,0,0,0,0]$} & {$[1,0,0,0,0,0]$} & {$[0,1,0,0,0,0]$} & {$[0,0,1,0,0,0]$} & {$[0,0,0,1,0,0]$} & {$[0,0,0,0,1,0]$} & {$[0,0,0,0,0,1]$} \\
\hline 0 & -77.8250 & -113.154 & -89.9610 & -111.006 & -123.422 & -423.253 & -105.896 \\
\hline 1 & 17.3950 & 48.0138 & 21.2373 & 24.1006 & 22.5338 & 69.0745 & 23.1594 \\
\hline 2 & -0.32512 & -4.25313 & -0.52500 & -0.01947 & -0.28432 & 37.9514 & -0.28038 \\
\hline 3 & 0.61222 & 3.65345 & 0.66457 & 0.87580 & 0.89884 & 70.3987 & 0.84611 \\
\hline 4 & -0.08144 & -0.60203 & -0.11427 & -0.10723 & -0.11883 & 109.611 & -0.11249 \\
\hline 5 & 0.03843 & 0.27888 & 0.04450 & 0.05607 & 0.06345 & 162.100 & 0.05742 \\
\hline 6 & -0.00812 & -0.02089 & -0.01284 & -0.01206 & -0.01483 & 221.697 & -0.01283 \\
\hline 7 & 0.00274 & -0.01044 & 0.00378 & 0.00482 & 0.00536 & 272.566 & 0.00458 \\
\hline 8 & -0.00067 & 0.00267 & -0.00130 & -0.00129 & -0.00147 & 284.240 & -0.00119 \\
\hline 9 & 0.00020 & 0.00249 & 0.00037 & 0.00044 & 0.00047 & 209.221 & 0.00037 \\
\hline 10 & -0.00005 & -0.00495 & -0.00013 & -0.00013 & -0.00013 & -13.5316 & -0.00010 \\
\hline 11 & 0.00001 & 0.00321 & 0.00004 & 0.00004 & 0.00004 & -437.751 & 0.00003 \\
\hline 12 & $<1.0 \mathrm{e}-05$ & -0.00096 & -0.00001 & -0.00001 & -0.00001 & -1063.53 & $<1.0 \mathrm{e}-05$ \\
\hline 13 & & -0.00045 & $<1.0 \mathrm{e}-05$ & $<1.0 \mathrm{e}-05$ & $<1.0 \mathrm{e}-05$ & -1755.78 & \\
\hline 14 & & 0.00081 & & & & -2130.55 & \\
\hline 15 & & -0.00059 & & & & -1442.26 & \\
\hline 16 & & 0.00022 & & & & 1440.04 & \\
\hline 17 & & 0.00002 & & & & 7798.50 & \\
\hline 18 & & -0.00011 & & & & 18320.1 & \\
\hline 19 & & 0.00009 & & & & 31555.9 & \\
\hline 20 & & -0.00004 & & & & 41458.9 & \\
\hline 30 & & $<1.0 \mathrm{e}-05$ & & & & $7.0 \mathrm{e}+06$ & \\
\hline$V C I[3]$ & 5778.89357 & 8562.57175 & 7525.56402 & 7278.85923 & 6925.23893 & 8570.99222 & 7021.58903 \\
\hline$n$ & {$[0,0,0,0,0,0]$} & {$[1,0,0,0,0,0]$} & {$[0,1,0,0,0,0]$} & {$[0,0,1,0,0,0]$} & {$[0,0,0,1,0,0]$} & {$[0,0,0,0,1,0]$} & {$[0,0,0,0,0,1]$} \\
\hline 0 & -77.8250 & -113.154 & -89.9610 & -111.006 & -123.422 & -423.253 & -105.896 \\
\hline 1 & -0.32512 & -4.25313 & -0.52500 & -0.01947 & -0.28431 & 37.9514 & -0.28038 \\
\hline 2 & 0.65030 & 7.47277 & 0.79050 & 1.01756 & 0.97215 & -18.9283 & 0.92571 \\
\hline 3 & -0.08616 & -0.65278 & -0.09434 & -0.09574 & -0.13365 & 0.40470 & -0.12207 \\
\hline 4 & 0.04417 & 0.52775 & 0.06421 & 0.07495 & 0.07741 & -3.68385 & 0.06999 \\
\hline 5 & -0.00947 & -0.23142 & -0.01307 & -0.01406 & -0.01876 & 0.23663 & -0.01580 \\
\hline 6 & 0.00354 & 0.12239 & 0.00598 & 0.00685 & 0.00761 & -0.67975 & 0.00644 \\
\hline 7 & -0.00091 & -0.02980 & -0.00155 & -0.00173 & -0.00224 & 0.07279 & -0.00176 \\
\hline 8 & 0.00030 & 0.01390 & 0.00066 & 0.00070 & 0.00081 & -0.12966 & 0.00064 \\
\hline 9 & -0.00008 & -0.00629 & -0.00020 & -0.00021 & -0.00026 & 0.01814 & -0.00019 \\
\hline 10 & 0.00003 & 0.00280 & 0.00008 & 0.00008 & 0.00009 & -0.02554 & 0.00006 \\
\hline 11 & $<1.0 \mathrm{e}-05$ & -0.00090 & -0.00003 & -0.00003 & -0.00003 & 0.00399 & -0.00002 \\
\hline 12 & & 0.00039 & 0.00001 & $<1.0 \mathrm{e}-05$ & $<1.0 \mathrm{e}-05$ & -0.00515 & $<1.0 \mathrm{e}-05$ \\
\hline 13 & & -0.00017 & $<1.0 \mathrm{e}-05$ & & & 0.00083 & \\
\hline 14 & & 0.00007 & & & & -0.00105 & \\
\hline 15 & & -0.00003 & & & & 0.00016 & \\
\hline 16 & & 0.00001 & & & & -0.00022 & \\
\hline 17 & & $<1.0 \mathrm{e}-05$ & & & & 0.00003 & \\
\hline 18 & & & & & & -0.00005 & \\
\hline 19 & & & & & & $<1.0 \mathrm{e}-05$ & \\
\hline$V C I[3]$ & 5778.89357 & 8562.57175 & 7525.56402 & 7278.85923 & 6925.23893 & 8570.99222 & 7021.58903 \\
\hline
\end{tabular}


TABLE II: Above difference between VMP $[n]$ (specific-state) and VCI[3] energies for overtones and combination bands of formaldehyde below $9000 \mathrm{~cm}^{-1}$. Below difference between VAPT $[n]$ (Møller-Plesset, specific-state) and VCI[3] energies for overtones and combination bands of formaldehyde below $9000 \mathrm{~cm}^{-1}$. Units are $\mathrm{cm}^{-1}$.

\begin{tabular}{|c|c|c|c|c|c|c|c|c|}
\hline$n$ & {$[0,0,0,2,0,0]$} & {$[0,0,0,0,0,2]$} & {$[0,0,0,1,0,1]$} & {$[0,0,1,1,0,0]$} & {$[0,0,1,0,0,1]$} & {$[0,1,0,1,0,0]$} & {$[0,1,0,0,0,1]$} & {$[0,0,2,0,0,0]$} \\
\hline 0 & -186.120 & -142.817 & -150.814 & -163.244 & -112.077 & -142.506 & -83.5495 & -152.181 \\
\hline 1 & 30.1863 & 32.7725 & 29.1230 & 31.0342 & 51.0603 & 28.3351 & 70.3343 & 28.7336 \\
\hline 2 & -4.42063 & -2.92391 & -0.25216 & 0.36042 & -22.3242 & -0.14398 & 64.7057 & 0.61480 \\
\hline 3 & 3.81575 & 1.77072 & 1.20583 & 1.33526 & 27.8968 & 1.06224 & 83.6440 & 1.43499 \\
\hline 4 & -2.17265 & -0.42062 & -0.16182 & -0.12988 & -17.7660 & -0.14367 & 97.2071 & -0.06769 \\
\hline 5 & 1.51633 & 0.07024 & 0.09190 & 0.09350 & -10.9940 & 0.07288 & 104.962 & 0.06840 \\
\hline 6 & -1.02863 & 0.14001 & -0.02264 & -0.01967 & 55.9833 & -0.02349 & 104.967 & -0.02234 \\
\hline 7 & 0.69190 & -0.21168 & 0.00858 & 0.00916 & -91.6704 & 0.00623 & 98.3409 & 0.00446 \\
\hline 8 & -0.45529 & 0.20985 & -0.00249 & -0.00254 & 65.4835 & -0.00304 & 88.2469 & -0.00280 \\
\hline 9 & 0.29252 & -0.17307 & 0.00083 & 0.00093 & 80.1019 & 0.00064 & 79.3476 & 0.00075 \\
\hline 10 & -0.18229 & 0.12537 & -0.00025 & -0.00029 & -339.675 & -0.00037 & 76.1124 & -0.00028 \\
\hline 11 & 0.10944 & -0.07995 & 0.00008 & 0.00010 & 543.908 & 0.00008 & 80.9085 & 0.00011 \\
\hline 12 & -0.06250 & 0.04298 & -0.00002 & -0.00003 & -309.849 & -0.00004 & 92.5405 & -0.00004 \\
\hline 13 & 0.03322 & -0.01632 & $<1.0 \mathrm{e}-05$ & 0.00001 & -787.668 & 0.00001 & 106.116 & 0.00002 \\
\hline 14 & -0.01565 & -0.00068 & & $<1.0 \mathrm{e}-05$ & 2659.71 & $<1.0 \mathrm{e}-05$ & 114.663 & $<1.0 \mathrm{e}-05$ \\
\hline 15 & 0.00565 & 0.00983 & & & -3870.52 & & 112.223 & \\
\hline 16 & -0.00039 & -0.01332 & & & 1283.99 & & 97.2749 & \\
\hline 17 & -0.00203 & 0.01317 & & & 8301.44 & & 74.8090 & \\
\hline 18 & 0.00283 & -0.01105 & & & -23160.3 & & 55.5100 & \\
\hline 19 & -0.00280 & 0.00816 & & & 29574.9 & & 51.5571 & \\
\hline 20 & 0.00239 & -0.00527 & & & -29.2365 & & 70.2133 & \\
\hline 30 & -0.00003 & 0.00025 & & & $1.9 \mathrm{e}+07$ & & 208.416 & \\
\hline 50 & $<1.0 \mathrm{e}-05$ & $<1.0 \mathrm{e}-05$ & & & $-8.3 e+11$ & & 7311.78 & \\
\hline$V C I[3]$ & 8076.92868 & 8266.22525 & 8171.96016 & 8418.32902 & 8507.38957 & 8662.08376 & 8719.99894 & 8778.30299 \\
\hline$n$ & {$[0,0,0,2,0,0]$} & {$[0,0,0,0,0,2]$} & {$[0,0,0,1,0,1]$} & {$[0,0,1,1,0,0]$} & {$[0,0,1,0,0,1]$} & {$[0,1,0,1,0,0]$} & {$[0,1,0,0,0,1]$} & {$[0,0,2,0,0,0]$} \\
\hline 0 & -186.120 & -142.817 & -150.814 & -163.244 & -112.077 & -142.506 & -83.5495 & -152.181 \\
\hline 1 & -4.42063 & -2.92391 & -0.25216 & 0.36042 & -22.3242 & -0.14398 & 64.7057 & 0.61480 \\
\hline 2 & 3.60077 & 2.09677 & 1.34225 & 1.57696 & 1.70111 & 1.30442 & 102.696 & 1.82030 \\
\hline 3 & -1.64449 & -0.75397 & -0.18565 & -0.10683 & -2.51403 & -0.09383 & 91.0549 & -0.06454 \\
\hline 4 & 1.09782 & 0.65867 & 0.11842 & 0.13975 & 0.30689 & 0.12941 & 91.7997 & 0.06465 \\
\hline 5 & -0.63080 & -0.30207 & -0.03002 & -0.02217 & -0.31631 & -0.01855 & 91.9419 & -0.00495 \\
\hline 6 & 0.40077 & 0.17694 & 0.01316 & 0.01534 & 0.05848 & 0.01476 & 91.7361 & 0.03297 \\
\hline 7 & -0.24151 & -0.10009 & -0.00410 & -0.00346 & -0.04470 & -0.00282 & 91.7028 & -0.01009 \\
\hline 8 & 0.15097 & 0.06422 & 0.00159 & 0.00187 & 0.01160 & 0.00195 & 91.7298 & -0.00342 \\
\hline 9 & -0.09236 & -0.03629 & -0.00054 & -0.00051 & -0.00725 & -0.00045 & 91.7186 & 0.00301 \\
\hline 10 & 0.05737 & 0.02183 & 0.00020 & 0.00024 & 0.00248 & 0.00028 & 91.7202 & 0.00121 \\
\hline 11 & -0.03529 & -0.01281 & -0.00007 & -0.00007 & -0.00139 & -0.00008 & 91.7205 & -0.00141 \\
\hline 12 & 0.02186 & 0.00777 & 0.00003 & 0.00003 & 0.00058 & 0.00004 & 91.7203 & -0.00004 \\
\hline 13 & -0.01348 & -0.00457 & $<1.0 \mathrm{e}-05$ & -0.00001 & -0.00031 & -0.00001 & 91.7203 & 0.00045 \\
\hline 14 & 0.00834 & 0.00274 & & $<1.0 \mathrm{e}-05$ & 0.00015 & $<1.0 \mathrm{e}-05$ & 91.7204 & -0.00006 \\
\hline 15 & -0.00514 & -0.00162 & & & -0.00008 & & 91.7204 & -0.00014 \\
\hline 16 & 0.00318 & 0.00097 & & & 0.00004 & & 91.7204 & 0.00005 \\
\hline 17 & -0.00196 & -0.00058 & & & -0.00002 & & 91.7204 & 0.00004 \\
\hline 18 & 0.00121 & 0.00035 & & & 0.00001 & & 91.7204 & -0.00002 \\
\hline 19 & -0.00075 & -0.00021 & & & $<1.0 \mathrm{e}-05$ & & 91.7204 & $<1.0 \mathrm{e}-05$ \\
\hline 20 & 0.00046 & 0.00012 & & & & & 91.7204 & \\
\hline 30 & $<1.0 \mathrm{e}-05$ & $<1.0 \mathrm{e}-05$ & & & & & 91.7204 & \\
\hline$V C I[3]$ & 8076.92868 & 8266.22525 & 8171.96016 & 8418.32902 & 8507.38957 & 8662.08376 & 8719.99894 & 8778.30299 \\
\hline
\end{tabular}


method. On the contrary, VAPT does not diverge for any state, in spite of the fact it fails in targeting one of the states.

Here we should also mention about the computational cost, which is lower per order for VMP due to the $2 n+1$ rule. As a rule of thumb one may compare $\operatorname{VMP}[2 n+1]$ against $\operatorname{VAPT}[n]$ which come at similar computational cost. In that comparison, VMP is clearly superior to VAPT for the non-troublesome states. Notwithstanding, it becomes evident that VAPT is capable of introducing more static correlation energy than $\mathrm{VMP}[2 n+1]$ despite $\operatorname{VAPT}[n]$ usually introduces less dynamic correlation energy. Prospective work faces the challenge to make VAPT yet computational cheaper, to reach similar cost to VMP methods.

\section{SUMMARY}

In this paper we introduce a new method in vibrational theory, the vibrational auto-adjusting perturbation theory (VAPT). VAPT is an alternative method to vibrational Rayleigh-Schrödinger perturbation theory (RSPT). The VAPT performs iteration steps with a twofold mechanism. First, a perturbative correction (by in principle any method, here we have chosen first order RSPT) is calculated, then, VAPT redefines the unperturbed system, and starts all over again. This last peculiarity is what makes VAPT capable of handling systems with significant static mode-correlation, avoiding the convergence problems suffered by VMP.

This feature is illustrated by calculation on the Fermi-resonant two-model system developed by Thompson and Truhlar and the quartic force field of Romanowski for formaldehyde. VAPT is shown to converge the series where VMP could not.

From this manuscript three main conclusions are drawn. First, VAPT is capable of introducing more static correlation than $\operatorname{VMP}[2 n+1]$. Second, $\operatorname{VAPT}[n]$ introduces less dynamic correlation energy than $\mathrm{VMP}[2 n+1]$, the method we should compare with to balance computational costs. Third, the major problem of our current implementation of VAPT is not divergence of VAPT, but the targeting of the correct state. Nevertheless, VAPT has targeting problems only for one case, which was one of the divergent VMP cases. In this sense, a faster VAPT method which avoids wrong targeting would be desired, so that we had a method that could effectively cope with static correlation and scale similar to VMP. Work in this line is in progress in our laboratories.

Overall, VAPT stands as a promising method in vibrational theory: it is easy to implement, it seems capable of handling resonant systems, and provides accuracy already for low order terms of its series.

\section{ACKNOWLEDGMENTS}

E.B. and J.M.B. thank the project CTQ2006-04410/BQU of the Spanish Ministerio de Ciencia y Tecnología. O.C. acknowledges support from the Danish Center for Scientific Computing (DCSC), the Danish national research foundation, the Lundbeck Foundation, and EUROHORCs for a EURYI award. J.M.L thanks the project CTQ200806696/BQU of the Spanish Ministerio de Ciencia y Tecnología.

\footnotetext{
* To whom the correspondence should be addressed: ematito@gmail.com

$\dagger$ To whom the correspondence should be addressed: josepm.luis@udg.edu

[1] M. Born and R. Oppenheimer, Ann. Phys. (Leipzig) 389, 457 (1927).

[2] J. Kongsted and O. Christiansen, J. Chem. Phys. 125, 124108 (2006).

[3] D. Toffoli, J. Kongsted, and O. Christiansen, J. Chem. Phys. 127, 204106 (2007).

[4] E. Matito, D. Toffoli, and O. Christiansen (submitted).

[5] J. M. Bowman, J. Chem. Phys. 68, 608 (1978).

[6] J. M. Bowman, Acc. Chem. Phys 19, 202 (1986).

[7] R. B. Gerber and M. A. Ratner, Chem. Phys. Lett. 195, 68 (1979).

[8] R. B. Gerber and M. A. Ratner, Adv. Chem. Phys. 97, 70 (1988).

[9] L. S. Norris, M. A. Ratner, A. E. Roitberg, and R. B. Gerber, J. Chem. Phys. 105, 11261 (1996).

[10] J. O. Jung and R. B. Gerber, J. Chem. Phys. 105, 10332 (1996).
} 
[11] O. Christiansen, J. Chem. Phys. 119, 5773 (2003).

[12] C. Møller and M. S. Plesset, Phys. Rev. 46, 618 (1934).

13] P. Seidler, M. B. Hansen, and O. Christiansen, J. Chem. Phys. 128 (2008).

[14] D. M. Benoit, J. Chem. Phys. 125, 244110 (2006).

[15] K. Yagi, S. Hirata, and K. Hirao, J. Chem. Phys. 127, 034111 (2007).

[16] O. Christiansen, J. Chem. Phys. 120, 2149 (2004).

[17] P. Seidler and O. Christiansen, J. Chem. Phys. 126, 204101 (2007).

[18] J. M. Bowman, K. M. Christoffel, and N. C. Handy, J. Phys. Chem. 83, 905 (1979).

[19] S. Carter, J. M. Bowman, and N. C. Handy, Theor. Chim. Acta 100, 191 (1998).

[20] K. M. Christoffel, J. M. Bowman, and N. C. Handy, Chem. Phys. Lett. 85, 220 (1982).

[21] O. Christiansen and J. M. Luis, Int. J. Quant. Chem. 104, 667 (2005).

[22] N. Matsunaga, G. M. Chaban, and R. B. Gerber, J. Chem. Phys. 117, 3541 (2002).

[23] V. Barone, J. Chem. Phys. 122, 014108 (2005).

[24] K. Yagi, S. Hirata, and K. Hirao, Phys. Chem. Chem. Phys 10, 1781 (2008).

[25] E. Besalú and R. Carbó-Dorca, J. Math. Chem. 21, 395 (1997).

[26] E. Besalú and R. Carbó-Dorca, Scientia gerundensis 23, 29 (1997).

[27] E. Besalú and J. M. Bofill, J. Comput. Chem. 19, 1777 (1998).

[28] T. C. Thompson and D. G. Truhlar, Chem. Phys. Lett. 75, 87 (1980).

[29] O. Christiansen, J. Chem. Phys. 120, 2140 (2004).

[30] J. K. G. Watson, Mol. Phys. 15, 479 (1968).

[31] J. K. G. Watson, Mol. Phys. 19, 465 (1970).

[32] A. Jäckle, G. Worth, H. D. Meyer, and M. H. Beck, Phys. Rep 324 (2000).

[33] O. Christiansen, Phys. Chem. Chem. Phys. 9, 2942 (2007).

[34] H. Romanowski, J. M. Bowman, and L. B. Harding, J. Chem. Phys 82, 4155 (1985).

[35] J. M. Barroso, Institute of Computational Chemistry. University of Girona, Girona (2008).

[36] O. Christiansen, M. B. Hansen, J. Kongsted, P. Seidler, D. Toffoli, M. Sparta, W. Győrffy, and E. Matito, University of Aarhus, Aarhus (2008). 